\title{
The IIHR Fluids Workshop, A Community of Scholars
}

James H. J. Buchholz

Department of Mechanical and Industrial Engineering

and IIHR - Hydroscience \& Engineering

The University of Iowa

james-h-buchholz@uiowa.edu

\begin{abstract}
A laboratory environment dedicated to undergraduate research and open-ended projects is described. The laboratory is designed to reduce barriers for student access to modern laboratory facilities, instruments and methods for research and open-ended projects, by providing dedicated laboratory resources, training and ongoing support through a community of peers. It is concluded that a plan for continued growth of the lab and increased impact on student learning will require simultaneous and coordinated developments in a number of interrelated areas including increasing student participation, establishing student management of lab functions, enhancing the technical capabilities of the lab, and securing sustained funding sources.
\end{abstract}

\section{Introduction}

Modern undergraduate engineering programs have changed dramatically in recent years through the introduction of new instructional technologies and research-based teaching methods. One of the objectives is to teach students to become life-long (or self-directed) learners. As discussed by Jiusto and DiBiasio ${ }^{2}$, success in developing life-long learners through undergraduate engineering education can be extraordinarily difficult to objectively and meaningfully assess. In light of the resulting ambiguities, they suggested that the best approach to developing life-long learners is to place students in environments that are expected to be conducive to developing skills needed for life-long learning. In addition, collaboration in learning has been shown to develop critical thinking skills through discussion, clarification of ideas, and evaluation of others' ideas ${ }^{4}$.

This paper discusses a laboratory environment established to enable primarily undergraduate experiential learning in an environment that fosters teaching and collaboration between peers. The primary objectives of the laboratory are to augment students' formal education with meaningful experiential learning opportunities, and to reduce barriers to student involvement in research activities. These objectives are being pursued through the implementation of two strategies. First, physical resources are developed, including experimental and computational facilities, and laboratory space for model construction and storage. Second, an infrastructure is being developed that is conducive to the formation of a strong community of undergraduate enthusiasts that fosters instruction, sharing of ideas, and collaboration.

This laboratory community, the IIHR Fluids Workshop, was established in the fall of 2012. Efforts made toward establishment of resources and the community that support the educational 
objectives are discussed here. In Section 2, the laboratory environment is described, while in Section 3, efforts to establish a viable student community are discussed. In an open-ended, multi-purpose environment populated by inexperienced researchers, establishing effective safety practices and a strong safety culture is paramount, and this effort is discussed in Section 4 . In Section 5, a brief summary of past and ongoing projects is reported. Section 6 discusses challenges and future directions of the lab.

\section{Laboratory Facilities}

The IIHR Fluids Workshop was established as an extension of the historic IIHR Fluids Lab - a state-of-the art teaching facility developed and supported by IIHR - Hydroscience \& Engineering, at the University of Iowa. The Fluids Lab contains several experiments and numerical facilities ${ }^{5}$ covering a range of fundamental topics in fluid dynamics, including investigations of the equations of motion, open channel flow, pipe flow, and forces on aerodynamic bodies. The apparatus and computational software tools provide demonstrations that support several fluids-related courses in the College of Engineering. In contrast, the Fluids Workshop is not designed with any particular study in mind. The goal of the Fluids Workshop is to make state-of-the art facilities, instrumentation and computational tools accessible for undergraduate open-ended projects and research.

The Fluids Workshop was established with support of the College of Engineering, IIHR Hydroscience \& Engineering, and the Departments of Civil and Environmental Engineering, and Mechanical and Industrial Engineering. Initial acquisitions of facilities and instrumentation for the lab have focused primarily on enabling visualization of flows since the study of fluid mechanics is highly visual, and visualization can promote an intuitive understanding of fluid flow to augment theoretical concepts discussed in undergraduate-level courses.

A 750 square foot laboratory space houses the Fluids Workshop, as shown in Figure 1a. The laboratory contains workbenches, a stocked tool cabinet, and several additional shelves and cabinets for storage of instrumentation, supplies, and student projects. A 50-inch monitor is also available for instruction and presentations.

Two flow facilities are used in the Fluids Workshop. A free-surface recirculating water channel

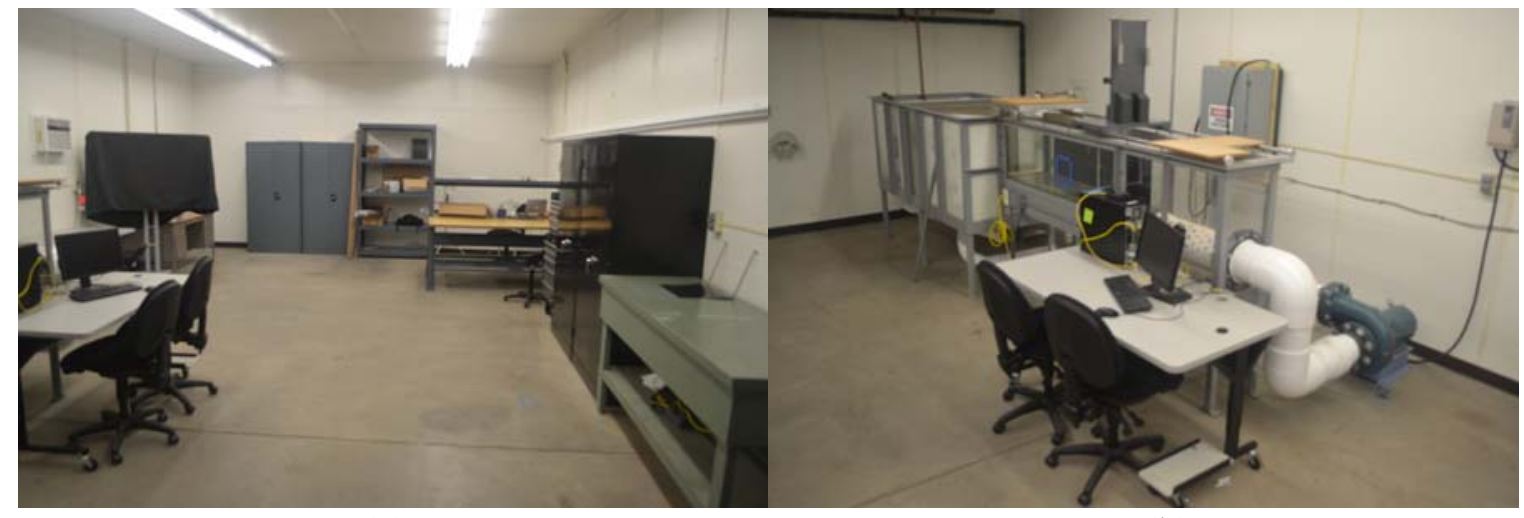

(a)

(b)

Figure 1: The Fluids Workshop. (a) The laboratory space, and (b) the free surface water channel. 
was designed and constructed specifically for the lab, as shown in Figure 1b, and a pre-existing recirculating open-jet wind tunnel is also used to support Fluids Workshop activities. The water channel has a test section of width $0.36 \mathrm{~m}$, depth $0.46 \mathrm{~m}$, and length $1.5 \mathrm{~m}$. The bed and side walls of the test section are glass to maximize optical access to the test section. The return plenum downstream of the test section is made from acrylic to allow optical access looking upstream into the test section. Flow conditioning consists of honeycomb flow straightener, three screens, and a three-dimensional contraction to maintain a highly uniform, low-turbulence flow in the test section so that high-quality studies can be conducted. Maximum flow velocity in the test section is approximately $1 \mathrm{~m} / \mathrm{s}$. In the design of the facility, the intention was for students to be able to conduct research of sufficient quality to present at scientific conferences or publish in peer-reviewed journals. The wind tunnel nozzle has a square cross section of $0.61 \mathrm{~m}$ on a side, and a maximum velocity of approximately $30 \mathrm{~m} / \mathrm{s}$. It is shared with the Fluids Lab.

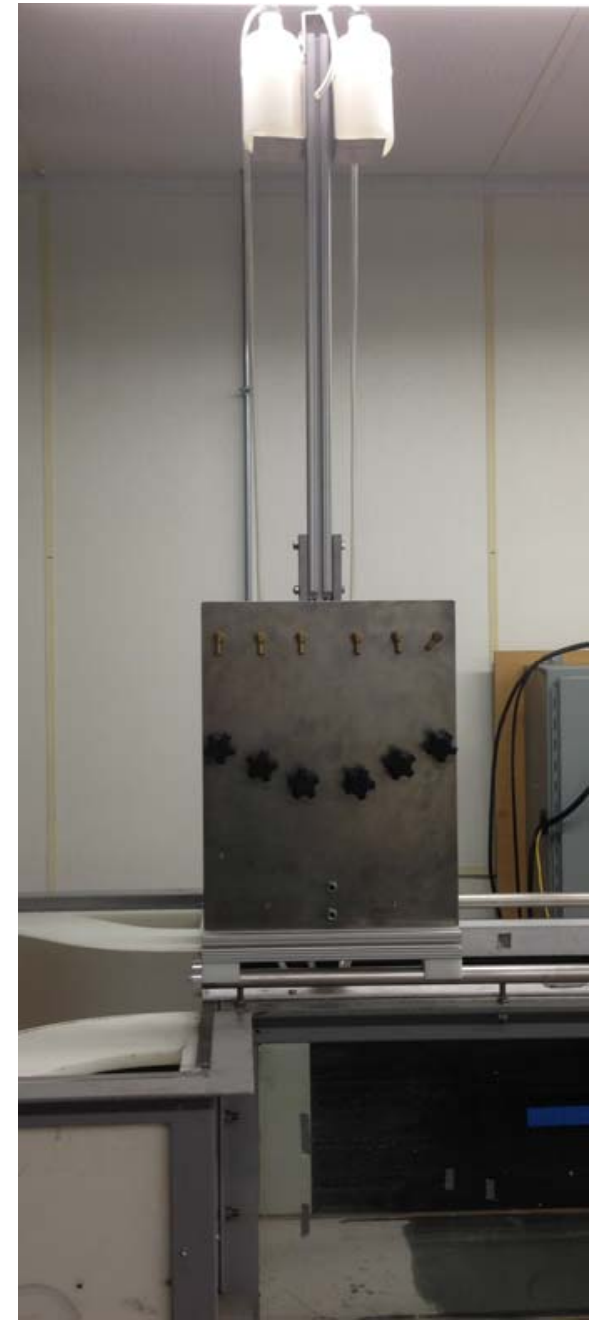

Figure 2: Dye injection system

An IDT NX-4 high-speed camera is used for qualitative dye visualization as well as particle image velocimetry (PIV) measurements. The camera is capable of acquiring 10-bit monochrome, 1-megapixel images at a rate of up to 2000 frames/second. Illumination is achieved using either LED lights or a 5-watt Laserglow diode-pumped solid state laser (532 nm) with light sheet optics, depending on the application. Dye injection for flow visualization is controlled using a gravity-fed 6-channel dye injection system shown in Figure 2. PIV measurements obtained using the camera and laser are currently analyzed using the open-source PIVlab software developed by W. Thielicke and E. J. Stamhuis ${ }^{6,7}$.

Complex motions of experimental models can be achieved using an integrated PID motion control system, consisting of a three-axis controller (Galil DMC-4030), for which linear and rotary motors are currently available on two axes. Thus, a variety of unsteady aerodynamic phenomena may be investigated. Three National Instruments LabVIEW-based data acquisition systems are also shared with the Fluids Lab, which can support a variety of instruments, shared with other laboratories, for measurement of quantities such as temperature and pressure.

An intuitive but powerful computational fluid dynamics flow solver is currently under development to complement the experimental facilities, in collaboration with Prof. H. S. Udaykumar and Prof. S. Vigmostad at the University of Iowa. The project, funded by Instructional Technology Services at the University of Iowa, involves development of a graphical user interface for the existing flow solver pELAFINT3D (parallel Eulerian Lagrangian Algorithm for Interfacial Transport in 3D) ${ }^{3}$. The Cartesian grid flow solver employs local mesh refinement, so that students do not need to 
deal with grid generation - a non-trivial task that can provide significant barriers for novice users.

\section{Development of a Student Community}

The student community environment is an important factor in reducing barriers to student involvement in research since a) peer instruction can significantly augment (and largely replace) instruction by faculty or graduate students who have limited time, b) participation in a local community of peers can make the lab a more inviting place to work, and c) discussion between laboratory participants can broaden students' exposure to ideas, and accelerate the development of new ideas as it does in the scientific community. A central strategy in the development of the laboratory has therefore involved establishing infrastructure that fosters community development.

Several initiatives are being pursued to encourage community development, which have so far resulted in isolated successes, as will be discussed in Section 6. Typically, 5 to 10 students have been participating in the laboratory in any given semester (with student numbers around the high end of the range during the summer). First, all students using the laboratory are required to submit a non-competitive online project application form. Some of the questions in the application form require students to describe their objectives, approach, and expected project outcomes in order to promote active engagement in their work. Other questions focus on what the student requires of the Fluids Workshop, including both physical resources and instruction (submission of the application form typically follows a discussion with the author where these aspects of the project are discussed). This provides a means to administratively pair students for the purpose of training, which immediately establishes a personal connection within the lab. To further facilitate development of such instructional relationships, all students participating in the Fluids Workshop are required to commit to contributing a resource back to the laboratory. This may consist of a broad range of products or services, including contribution of hardware or software developed in the course of their work within the Fluids Workshop, authoring a tutorial or reference document, or committing to training of a new student on use of an apparatus or method with which they have gained expertise.

Another approach recently taken to foster community growth is the establishment of laboratory meetings two to three times per week (in summer months when student productivity is high). In that experiment, the author led one of the weekly meetings, and the students met without faculty participation in at least one meeting per week. In the latter case, they were given a generic agenda designed to encourage students to explain their work to each other, discuss challenges, and to promote group participation in discussion of ideas and solutions to problems. For each meeting, two students were selected to chair the meeting, and to take minutes, which were reported back to the author. This strategy has so far been implemented only on a very preliminary basis, and there are not yet sufficient data to indicate whether the meetings have contributed significantly to the development of the community or the quality of the student projects. 


\section{Laboratory Safety}

From the perspective of student safety, the Fluids Workshop has several important differences in comparison to a traditional teaching laboratory. Students sometimes work unsupervised in the lab - often for the first time in an open-ended research environment - and, since the nature of student work can vary considerably, there is the potential to introduce new hazards with each project. In addition, the use of a class 4 laser in some projects introduces additional, substantial risks. It is therefore particularly important to establish sound safety policies and procedures and to foster a safety culture within the community so that new hazards can be identified.

In a recent report, the Committee on Establishing and Promoting a Culture of Safety in Academic Laboratory Research (National Research Council of the National Academies) made several recommendations for developing an effective safety culture within an institution, including incorporation of non-punitive incident and near-miss reporting as part of their safety cultures, and implementation of actions and activities to complement initial, ongoing, and periodic refresher training ${ }^{1}$. They define a safety culture as a "culture of habitual risk assessment, experiment planning, and consideration of worst-case possibilities", and they note that, "a strong, positive safety culture arises not because of a set of rules, but because of a commitment to safety throughout an organization." It was noted that academic researchers (e.g. graduate students and post docs) can be resistant to reporting incidents or raising safety concerns since they could result in delays or complications that could potentially jeopardize their education or financial security. Given the relatively smaller stakes in an undergraduate laboratory where the activities are a smaller contribution to the students' overall academic program, the Fluids Workshop is potentially a valuable resource for establishing good habits in students before they take on more advanced roles in other labs.

Several initiatives have been taken to promote safety in the lab. IIHR safety infrastructure is leveraged, which defines classes of laboratory users based on the inherent risks associated with their project and environment. Laser safety is of primary importance since biohazardous materials are not used, and chemical hazards are minimal in the lab (to date, the only chemicals permitted in the laboratory consist of dyes for flow visualization -- fluorescein and rhodamine B - and household adhesives and lubricants). A Level 0 classification is defined specifically for the Fluids Workshop, which specifies users who are performing work associated with the lab, but are not actually working within the laboratory environment (i.e. they are not subject to laboratory hazards). Level 1 users are defined as those who access laboratory spaces in which class $3 \mathrm{~b}$ or 4 lasers are in use. Most users are Level 1, and they are not permitted to operate lasers. Level 2 users are authorized to operate a laser. Finally, Level 3 users can operate lasers and/or use hazardous substances. So far there have not been any Level 3 users associated with the laboratory, as introduction of hazardous substances into the laboratory is to be avoided. It is unlikely that Level 3 users will ever be included in the Fluids Workshop, as the associated expertise and procedures are better handled in faculty research labs.

In order to be authorized for laboratory access, students must successfully complete required online safety courses administered by the Environmental Health and Safety office at the University of Iowa, and confirm completion of other requirements by signing a manifest. They are then entered into a database of laboratory users according to their safety classification. Students classified as Level 1 are required to take online safety courses on laser safety and 
personal protective equipment administered by the Environmental Health and Safety office at the University of Iowa, and to review customized documentation on laser safety. In addition to completion of the Level 1 requirements, Level 2 users must review standard operating procedures associated with the laser to be used, as well as the operating and safety instructions furnished by the laser manufacturer. Personal, hands-on training on safe laser operation by either the author or a designated experienced user (typically a graduate student) is also required.

Efforts to encourage a safety culture within the lab include periodic visits to the lab by the author in which the author participates in ongoing activities, emphasizing potential risks and safety strategies. Students are reminded to be attentive to potential laboratory hazards and report them immediately. In the required exit survey, students are also asked to describe any potential hazards observed in the lab, and to make recommendations for improvements.

Several standard policies have also been implemented in the laboratory, including prohibition of

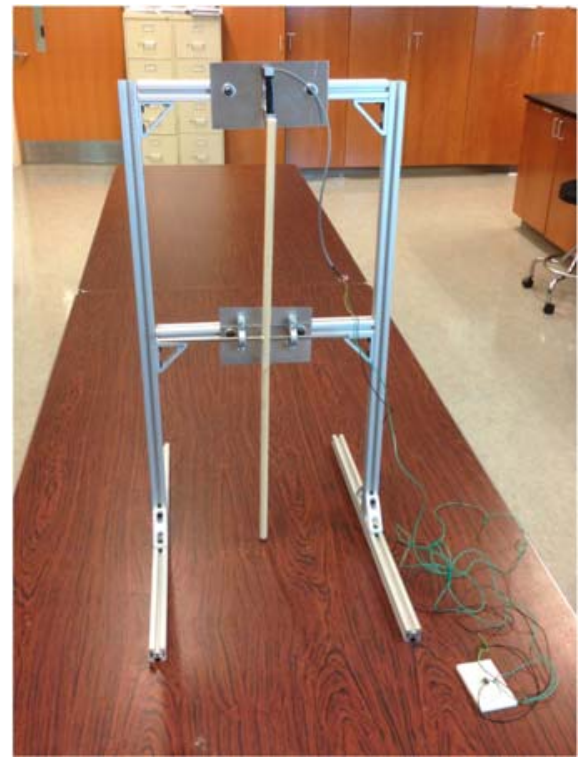

(a)

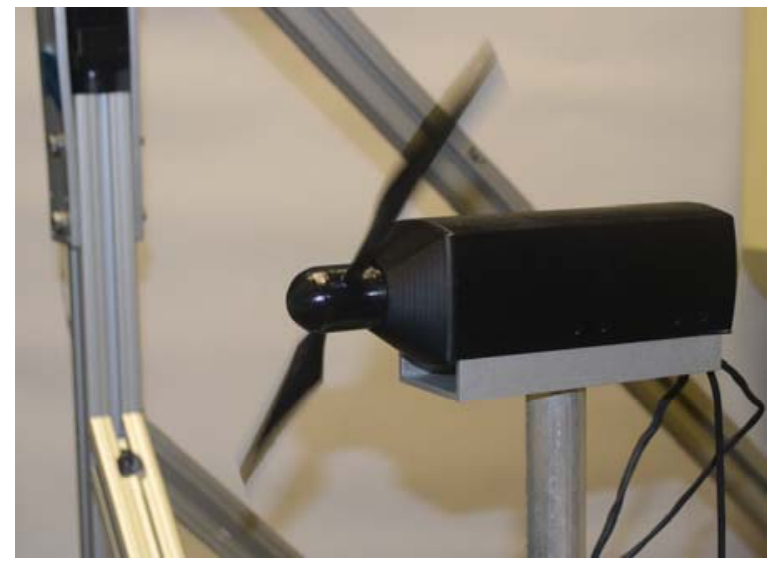

(b)

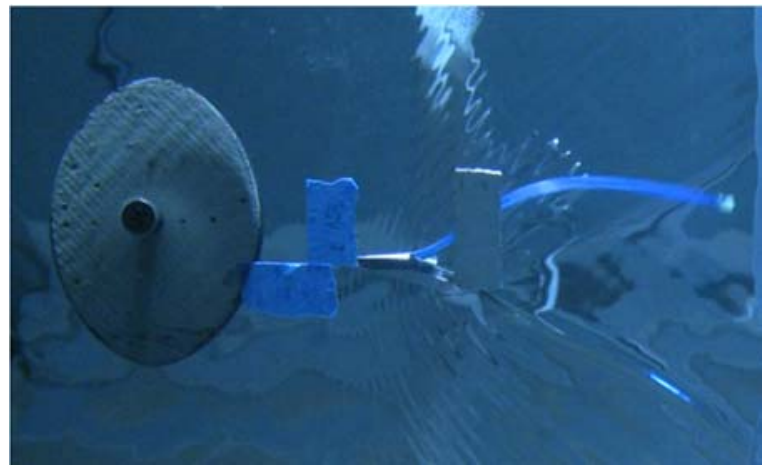

(c)

Figure 3: Example student projects. (a) single axis, variable-sensitivity force balance developed for the Fluids Workshop, (b) performance testing of a model wind turbine, and (c) energy harvesting from a bluff body wake. 
food and drink, working alone after normal business hours, and introduction of new chemicals into the lab without prior permission. Proper attire is required, including closed-toe shoes, and use of personal protective equipment supplied in the lab.

\section{Projects}

To date, the Fluids Workshop has supported activities within several courses, including ME:4080 Experimental Engineering, BME:4920 Biomedical Engineering Senior Design, ME:4125 Biomimetic Fluid Dynamics (developed and taught by the PI), and CEE:5372/ME:5162 Experimental Methods in Fluid Mechanics and Heat Transfer. Several undergraduate independent research projects have also been supported, with sponsors including the Iowa Center for Research by Undergraduates, the Secondary Student Training Program at the University of Iowa's Belin Blank Center (an international program providing research opportunities for gifted high school students), Iowa NSF EPSCoR (Research Experiences for Undergraduates), and the Iowa Space Grant Consortium sponsored by NASA.

Specific examples of projects conducted in the lab include:

- Design, construction, and implementation of a variable-sensitivity single-axis force balance for use in the Fluids Workshop (ME:4080). The device is shown in Figure 3a.

- Design and performance measurement of a model wind turbine (REU summer research). See Figure 3b.

- Study of flow in an aortic model with an aneurysm (CEE:5372).

- Development of an apparatus to investigate tadpole escape response (Iowa Space Grant, in collaboration with Prof. B. Fritzsch in the Department of Biology).

- Energy harvesting from bluff body wakes (independent research supported by the Iowa Center for Research by Undergraduates), as shown in Figure 3c.

- Flow dynamics on flapping wings and rotating blades.

\section{Challenges, and Plans for Further Development and Evaluation of Laboratory Impact}

Over the past two years, the nascent IIHR Fluids Workshop has provided opportunities, resources, and mentoring for dozens of students engaged in research and open-ended course projects within the College of Engineering and beyond. In exit surveys, virtually all students have also reported meaningful interactions with other students in the course of their projects, although, many of these relationships were pre-existing (e.g. student groups using the Fluids Workshop to complete a course project). Future viability and increased impact of the laboratory will rely heavily on growth of the student community for several reasons. Since management of the laboratory requires a considerable effort, given a constant flux of relatively inexperienced and otherwise preoccupied students, a long-term strategy for laboratory management must be developed, which will likely require the establishment of student officer positions similar to those established in student chapters of professional organizations and honor societies. A critical mass of committed, mature, and experienced undergraduate students will be required to achieve such structure, which would perform roles such as organizing laboratory clean-up efforts, coordinating mentoring activities and equipment sharing, and monitoring laboratory safety (e.g. promoting a safety culture). One possible source of leadership in this area is formal involvement 
of engineering honor societies, which typically include community service and high intellectual standards in their mandates.

Most of the projects conducted so far have involved senior undergraduate students who, being new to the lab at the beginning of their work, are not prepared or qualified to contribute knowledge to other users or undertake administrative roles - at least not without an established precedent - and then graduate before they have an opportunity to provide these services. It is therefore necessary to target students earlier in their academic program, and to enhance integration with college courses and student organizations such as to increase the opportunities for sustained involvement throughout a student's undergraduate program. A number of possible strategies to achieve this are being pursued, including:

1. Increased exposure of the lab to students, student organizations, and faculty in order to promote awareness of the lab resources, and to promote discussion of strategies to better serve college needs.

2. Expansion of laboratory capabilities to better serve the needs of a broader range of courses.

3. The establishment of a student-authored wiki discussing use of equipment, research methods, lab safety, etc. Since the wiki would be accessible for reading and writing by all lab participants, contributions to the collective knowledge of the group could be made with greater ease, and barriers to dissemination within the community, reduced.

4. Solicitation of small, funded industry projects to be completed by undergraduate students in the Fluids Workshop.

Regarding Strategy 3, there is evidence to suggest that user-authoring of content can enhance their engagement since the presence of a real audience promotes more thoughtful communication $^{8,9}$. Similarly, Wheeler et al. reported evidence that students developed critical thinking and writing skills through course-related wiki development; however, student concerns over ownership of ideas appeared to be more problematic than for more anonymous venues such as Wikipedia ${ }^{8}$.

Strategy 4 must be interwoven with the other strategies to enhance the impact on student learning and lab viability, and is not entirely new to the lab since some of the curricular design projects previously conducted within the lab have been sponsored by industry. Attracting several small industry projects to the lab will provide opportunities (and financial incentives) for students to engage in the lab, as well as funding for maintenance and expansion of lab capabilities. If managed effectively, it will also provide industry with inexpensive, low-risk opportunities for conducting testing and research, as well as opportunities to scout potential future employees. However, the viability of the program would likely depend on the efficacy of the lab to quickly train, and effectively support students as they pursue the projects. This will be most easily achieved with a larger community of students that can better provide peer education.

Assessment efforts of lab impact on student learning have so far consisted of fairly rudimentary measurements of student numbers, courses impacted, and information about student interactions in the lab collected from student exit surveys. Deeper questions about the impact of the lab on career opportunities and decisions, and on students' propensity for lifelong learning are more involved, and means of data collection and assessment are under consideration. 


\section{Conclusions}

In the past two years, the IIHR Fluids Workshop has provided opportunities for dozens of undergraduate students to engage in a safe and accessible laboratory environment in which they can pursue independent research and course-related open-ended projects. Dedicated space and equipment, and integration into a community of peers are two foundations of the lab. Experience so far suggests that a larger community is needed to support long-term viability and efficacy of the lab in supporting student learning, which will also increase the impact of the lab on the teaching mission of the university. It is proposed that this growth be concurrent with the development of self governance within the community, and the introduction of funded projects to increase opportunities for participation and to support the maintenance and growth of the lab. Such services provided by the lab will be enhanced by a stronger student community so that growth will require coordination of many facets of the lab.

\section{Acknowledgments}

Establishment of the IIHR Fluids Workshop has been supported by the College of Engineering, IIHR - Hydroscience \& Engineering, and the Departments of Civil and Environmental Engineering and Mechanical and Industrial Engineering at the University of Iowa.

\section{Bibliography}

1. Committee on Establishing and Promoting a Culture of Safety in Academic Laboratory Research (2014). Safe Science: Promoting a Culture of Safety in Academic Chemical Research. National Research Council of the National Academies, The National Academies Press, Washington, D.C.

2. Jiusto, S. and DiBiasio, D. (2006). Experiential learning environments: do they prepare our students to be selfdirected, life-long learners? Journal of Engineering Education 95, pp. 195-204.

3. Krishnan, S., Marella, S., and Udaykumar, H. S. (2009) Adaptively refined, parallelized, sharp interface Cartesian grid method for three-dimensional moving boundary problems. International Journal on Computational Fluid Dynamics 23, pp. 1-24.

4. Sanders, M. (1995). Collaborative learning enhances critical thinking. Journal of Technology Education 7(1). http://scholar.lib.vt.edu/ejournals/JTE/v7n1/gokhale.jte-v7n1.html?ref=Sawos.Org

5. Stern, F., Xing, T., Yarbrough, D. B., Rothmayer, A., Rajagopalan, G., Otta, S. P., Caughey, D., Bhaskaran, R., Smith, S., Hutchings, B., and Moeykens, S. (2006) Hands-on CFD educational interface for engineering courses and laboratories, Journal of Engineering Education 95(1), pp. 63-83.

6. Thielicke, W. (2014). The Flapping Flight of Birds - Analysis and Application. Ph.D. Thesis, Rijksuniversiteit Groningen. 
7. Thielicke, W. and Stamhuis, E. J. (2014). PIVlab - Time-Resolved Digital Particle Image Velocimetry Tool for MATLAB. http://dx.doi.org/10.6084/m9.figshare.1092508.

8. Wheeler, S., Yeomans, P., and Wheeler, D. (2008). The good, the bad and the wiki: evaluating student-generated content for collaborative learning. British Journal of Educational Technology 39(6), pp. 987-995.

9. Williams, J. B. and Jacobs, J. (2004). Exploring the use of blogs as learning spaces in the higher education sector. Australasian Journal of Educational Technology 20(2), pp. 232-247. 